\title{
Full numerical simulations of multifluid flows
}

Grétar Tryggvason* and S. Ozen Unverdi

Department of Mechanical Engineering and Applied Mechanics, The University of Michigan, Ann Arbor, Michigan 48109-1120

(Received 28 August 1990)

To fully understand the behavior of multifluid systems, one must have good insight into the basic micromechanisms that govern the evolution of a single "structure" (e.g., a bubble or a drop) and the interactions of a few such basic entities. In addition to the usual questions about the relative magnitude of the various physical effects (inertia, viscosity, and surface tension) for multifluid systems, the effects of surface phenomena such as contaminants must be addressed. Full numerical simulations are, in principle, ideally suited to provide this information. Not only are all the quantitative data readily available, but various physical processes can be turned on and off at will. In practice, however, simulations of multifluid problems are one of the difficult areas of computational fluid dynamics. Almost all current studies of multifluid problems make a number of simplifications, such as inviscidness, Stokes flow, two-dimensionality, or axisymmetry. Although such models capture some of the important behavior, they often put severe constraints on the problems that can be investigated. Many of the fundamental processes in multifluid flow involve fully three-dimensional flows, where both inertia and viscous effects must be accounted for. To address these effects, we have recently developed a front-tracking method for multifluid, incompressible flows that appears to be both accurate and robust. The method has been implemented for both two- as well as fully three-dimensional situations. In this paper, we will discuss two problems that we are currently investigating using this numerical method: the Rayleigh-Taylor instability and the motion of bubbles and drops. For fluid mixing induced by unstable stratification, the Rayleigh-Taylor instability where a heavy fluid falls into a lighter underlying fluid, is the prototypical example. Indeed, for such flows its importance is similar to that of the Kelvin-Helmholtz instability for fluid mixing induced by a shear flow. For small density stratification, we show that threedimensionality can lead to a large amplitude vortex structure that differs considerably from what two-dimensional simulations predict. The different vortical configuration leads to more rapid nonlinear growth for the fully three-dimensional case, even though the linear growth rate is the same. We also show how viscosity stratification modifies the evolution. For the weakly stratified case, where inviscid calculations predict symmetric evolution with respect to the heavy and the light fluid, viscosity stratification leads to considerable asymmetry, with the more viscous fluid forming big round bubbles and the less viscous one being confined to narrow fingers. The effect of density stratification for viscous three-dimensional motion will also be discussed. For many mixing problems, the long-time state consists of a dispersed phase that forms drops or bubbles in another phase. We will discuss preliminary investigations of such flows. Calculations of rising bubbles for various values of surface tension and viscosity (both in two and three dimensions) appear to correlate well with experimental observations and steady-state calculations in the literature. The interactions of bubbles with each other, density interfaces, and vortices will also be discussed. 